\title{
Changes in Human Muscle Spindle Sensitivity during a Proprioceptive Attention Task
}

\author{
Valérie Hospod, Jean-Marc Aimonetti, Jean-Pierre Roll, and Edith Ribot-Ciscar \\ Neurobiologie Intégrative et Adaptative, Aix-Marseille Université, Centre National de la Recherche Scientifique, Unité Mixte de Recherche 6149, 13331 \\ Marseille Cedex 03, France
}

The aim of the present study was to test whether fusimotor control of human muscle spindle sensitivity changed when attention was selectively directed to the recognition of an imposed two-dimensional movement in the form of a written symbol.

The unitary activities of 32 muscle spindle afferents ( $26 \mathrm{Ia}, 6 \mathrm{II}$ ) were recorded by microneurography at the level of the common peroneal nerve. The patterns of firing rate in response to passive movements of the ankle, forming different letters or numbers, were compared in two conditions: control and recognition. No visual cues were given in either condition, but subjects had to recognize and name the character in one condition compared with not paying attention in the control condition.

The results showed that $58 \%$ of the tested Ia afferents presented modified responses to movements when these had to be recognized. Changes in Ia afferent responses included decreased depth of modulation, increased variability of discharge, and changes in spontaneous activity. Not all changes were evident in the same afferent. Furthermore, the percentage of correctly recognized movements amounted to $63 \%$ when changes were observed, but it was only $48 \%$ when the primary ending sensitivity was unaltered. The responses of group II afferents were only weakly changed or unchanged. It is suggested that the altered muscle spindle sensitivity is because of selective changes in fusimotor control, the consequence of which might be to feed the brain movement trajectory information that is more accurate.

Key words: fusimotor neurons; muscle spindle; noise; kinesthesia; proprioception; microneurography

\section{Introduction}

Kinesthesia relies on sensory feedback from various types of mechanoreceptors in the skin, joints, and muscles (Gandevia and Burke, 1992). Among these sensory sources, muscle proprioceptive information plays a major role. Indeed, sensations of illusory movements are induced by the application of mechanical vibrations to muscle tendons, which are known to preferentially drive muscle spindle primary endings (Goodwin et al., 1972; Roll and Vedel, 1982; Roll et al., 1989).

Muscle proprioceptive information may be modulated by the behavioral context. This modulation is directly performed by the CNS, which can change static and/or dynamic sensitivity of muscle spindles through selective control of static and/or dynamic $\gamma$ fusimotor neurons (for review, see Hulliger, 1984; Prochazka, 1996). In the conscious cat, it has been shown that the response characteristics of spindle afferents would be chosen by the CNS to be appropriate for the type of movement performed. More precisely, the fusimotor action would switch from largely static during predictable and stereotyped movements, such as stepping, to largely dynamic during unpredictable or novel motor activities,

Received Feb. 9, 2007; revised April 13, 2007; accepted April 16, 2007

This work was supported by the Association Française contre les Myopathies and by Centre National de la Recherche Scientifique grants. We thank Dr. Benjamin Mattei for developing the program under Matlab software.

Correspondence should be addressed to Edith Ribot-Ciscar, Neurobiologie Intégrative et Adaptative, AixMarseille Université, Centre National de la Recherche Scientifique, Unité Mixte de Recherche 6149, Pôle 3C Case B, Centre de St. Charles, 3 Place Victor Hugo, 13331 Marseille Cedex 03, France. E-mail: Edith.Ribot-Ciscar@up.univ-mrs.fr.

DOI:10.1523/JNEUROSCI.0572-07.2007

Copyright $\odot 2007$ Society for Neuroscience $\quad$ 0270-6474/07/275172-07\$15.00/0 such as imposed movements (Prochazka et al., 1985). Also, for the decerebrate cat during fictive locomotion, Taylor et al. (2000) showed that different types of intrafusal fibers are separately controlled, suggesting that the "CNS may adapt the properties of the muscle spindle to suit different functional situations."

In humans, it was originally considered that the main role of the fusimotor system was to prevent the silencing of muscle spindle endings during muscle shortenings, because changes in muscle spindle sensitivity often correlate with changes in muscular activities ( $\alpha-\gamma$ coactivation) (for review, see Vallbo et al., 1979; Prochazka, 1996). However, the fusimotor system may also allow task-dependent parametric control of muscle spindle feedback. This is supported by our previous study showing selective changes in muscle spindle sensitivity during the execution of a mental computation task (Ribot-Ciscar et al., 2000). These last observations, on completely relaxed subjects, suggest that the CNS retains the potential to selectively control muscle spindle sensitivity.

Herein, we investigated whether such a control might be involved in an attention task that forces the subject to rely on proprioceptive cues only. We compared the response of muscle spindle afferents to two-dimensional (2D) movements imposed while the subject was instructed not to pay attention to or to focus on the movement trajectory to recognize and name it, both in the absence of visual feedback. Because the movements had to be sufficiently complex to involve the subject's attention and yet remain easy to name, we chose movement trajectories forming letter or number shapes, recently shown to give rise to a precise 

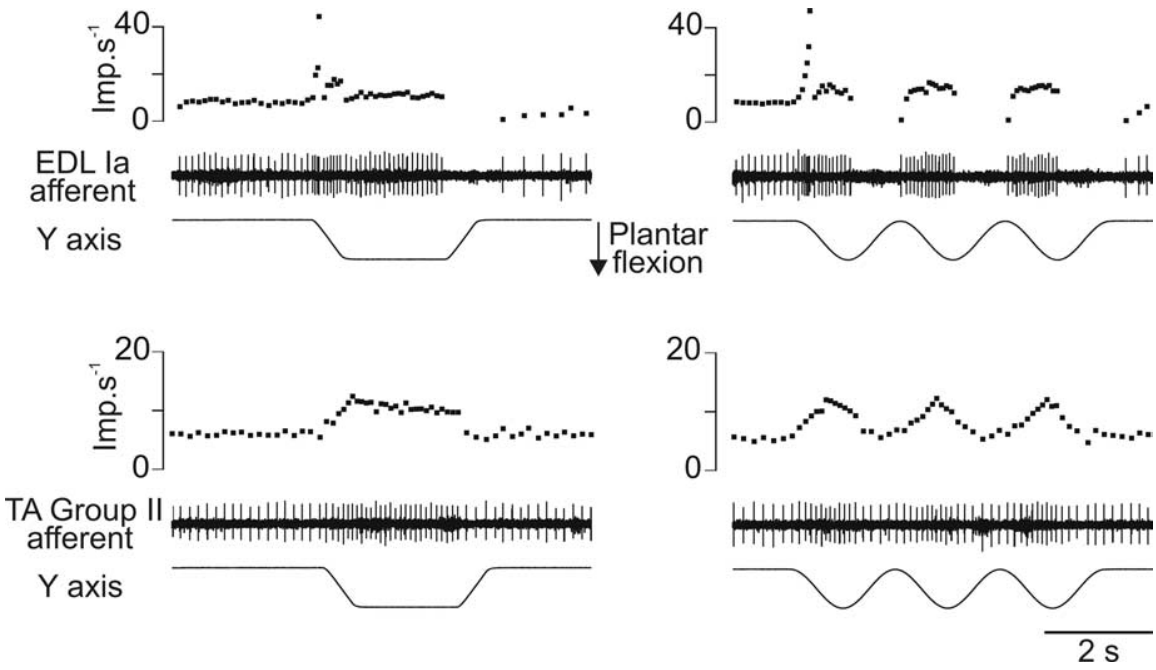

Figure 1. Examples of responses to ramp, hold (left), and sinusoidal (right) movements used to classify the afferent as primary (upper part) or secondary (lower part). Each part of the figure panels represents, from bottom to top, the imposed movement, the unitary activity, and the corresponding instantaneous discharge frequency curve. Imp, Impulse.

classify the unit, the sensitivity to muscle tendon vibration $(10-100 \mathrm{~Hz}$ ) was also tested (Roll et al., 1989).

The absence of any muscle activity was controlled throughout the experiment by recording surface electromyographic (EMG) activity. Two pairs of surface electrodes were placed over the TA and PL muscles. The interelectrode distance was $4 \mathrm{~cm}$. The EMGs were recorded with high gain $(10,000)$ and a bandpass of 3-3000 $\mathrm{Hz}$. They were sampled at $10 \mathrm{kHz}$.

In addition, because fusimotor activity may be triggered by environmental, behavioral, or cognitive factors (Ribot et al., 1986), the subject's level of arousal was monitored throughout the experiment by recording electrodermal activity (Critchley, 2002). The movements were imposed in control trials only when the electrodermal activity recording was flat. It should be specified that the electrodermal activity recording generally tended to become bumpy in the recognition situation. However, it could also stay almost flat depending on the subject and trials, even when the subject succeeded in recognizing the movement. For that reason, this monitoring appeared unsuitable for quantify-

and reproducible muscle proprioceptive signature (Roll et al., 2004; Albert et al., 2005).

The results show that more than half of the Ia afferents exhibited changes in their responses to movements when these had to be recognized compared with the situation in which no attention was paid. The changes observed were a decrease in the depth of the modulation of discharge associated or not with increased interspike variability. The responses of group II afferents were only weakly changed or unchanged. The nature of the fusimotor effects and their functional significance are discussed.

\section{Materials and Methods}

Experiments were performed on 26 healthy volunteers (mean age 22 years; range, 20-23), all of whom gave their written informed consent to the experimental conditions, as required by the Declaration of Helsinki. This study was approved by the local ethics committee (Comité Consultatif de Protection des Personnes se prêtant à des Recherches Biomédicales, Marseille I). The subjects were selected on the basis of their ability to relax. The activity of single muscle spindle endings originating from tibialis anterior (TA; $n=9$ ), extensor digitorum longus (EDL; $n=15$ ), extensor hallucis longus $(n=3)$, and peroneus lateralis (PL; $n=5)$ muscles were recorded from the common peroneal nerve by the microneurographic technique (Vallbo and Hagbarth, 1968; Bergenheim et al., 1999).

Data recordings. Muscle spindle afferent activity was recorded using an insulated tungsten microelectrode (impedance $300 \mathrm{k} \Omega$ to $1 \mathrm{M} \Omega$ tested at $1 \mathrm{kHz}$; tip diameter, $\sim 5-8 \mu \mathrm{m}$; length, $30 \mathrm{~mm}$; Frederick Haer, Bowdoinham, ME) inserted into the common peroneal nerve at the popliteal fossa level. Recordings were continuously monitored on an oscilloscope and a loudspeaker. Neural activity was amplified $(100,000 \times)$, using a bandpass of $300-3000 \mathrm{~Hz}$ to ensure an optimal signal-to-noise ratio and sampled at a $20 \mathrm{kHz}$ frequency.

Recordings were made from a total of 32 muscle spindle afferents; 26 were classified as primary and six as secondary afferents. This classification depended on their irregular or regular spontaneous activity, their high/low dynamic sensitivity, and silence/activity during muscle shortenings (Edin and Vallbo, 1990). The two latter characteristics were deduced from the response to passive ramp and hold movements as well as to sinusoidal movements (Fig. 1) imposed in the "preferred sensory direction" of the receptor-bearing muscle (i.e., $90^{\circ}$ and $311^{\circ}$ for afferents belonging to dorsal flexor and PL muscles, respectively) (Bergenheim et al., 2000). When these identification criteria did not allow us to clearly ing the subject's involvement in the task. It was therefore used only to ensure, as far as possible, that the subject was mentally relaxed in the control session to favor a $\gamma$-free condition. This recording was performed using two surface electrodes placed on each side of the left hand (gain, 500; bandpass, 0.1-100 Hz; sampling frequency, $100 \mathrm{~Hz}$ ).

Experimental setup. The subject was comfortably seated in an armchair, with the legs positioned in cushioned grooves so that a standardized relaxed position could be maintained without any muscle activity occurring. The knee joint was at an angle of $\sim 120-130^{\circ}$, and the feet were rested on supports. The right foot was laid on a stationary plate and the left foot was attached to a rotating pedal connected to a computercontrolled machine.

This machine was specially designed for our research aims (for a detailed description, see Bergenheim et al., 2000). It enabled imposing 2D movements on the ankle joint so that the tip of the foot made different number shapes $(1,2,3,6,8,9)$ or letters (a, b, e, l, m) and a word (in). This word was a "trap" to increase the difficulty of the recognition task.

These various shapes were written by the experimenter on a digitizing tablet before the experimental series and then transferred to the computer-controlled machine (Roll et al., 2004). These writing movements were identical in each experiment. The scale of all of these trajectories was adapted to fit a $50 \times 80 \mathrm{~mm}$ displacement frame around the tip of the foot. Their velocities varied during each movement in a "natural" way, reproducing the continuously varying writing velocity of the experimenter when writing on the digitizing tablet. The $x$ and $y$ components of each movement were sampled at $100 \mathrm{~Hz}$.

Experimental protocol. The unitary muscle spindle responses to writing movements were recorded in two situations: (1) when the subject received the instruction not to pay attention to the movement (i.e., "control situation"); (2) when the subject's attention was focused on the movement trajectory to recognize and verbally name it (i.e., "recognition situation"). In both situations, the subjects were required to keep their eyes closed.

In the control condition, a series of movements was chosen. The set of "letters" was preferentially chosen, and the set of "numbers" was used when a subject had previously been exposed to the set of letters and could therefore have become familiar with these movements. In this control situation, we waited for the electrodermal activity to be flat before starting the imposition of movements.

For each set and in each situation, the movements were imposed in random order. The first movement was started by the experimenter, and $2 \mathrm{~s}$ after its completion, the machine automatically repositioned the foot to the start position of the following writing movement (see Fig. 2). The 


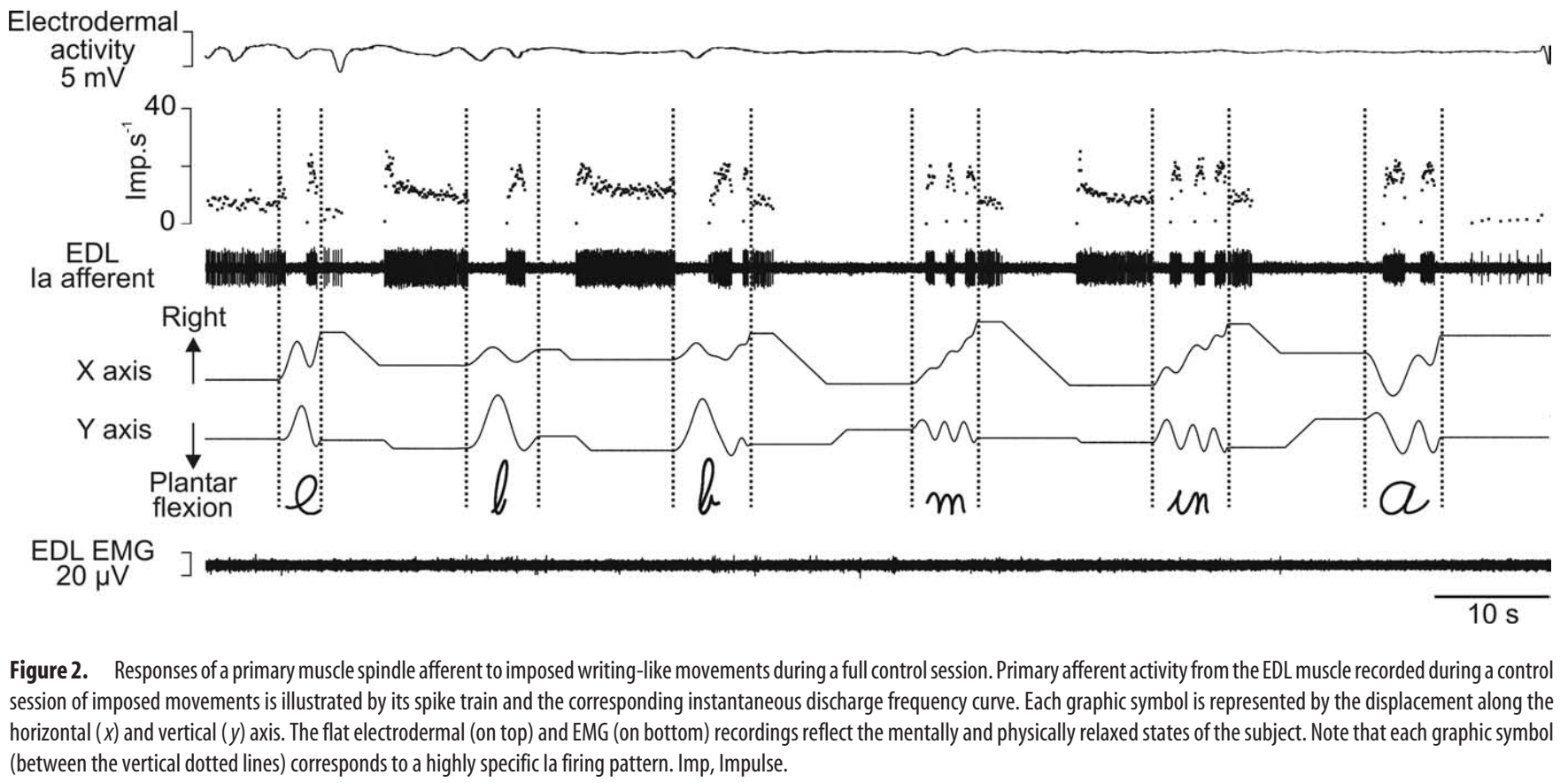

experimenter started the following movement according to the presence or absence of spontaneous activity of the afferent under consideration. More precisely, when a spontaneous activity was triggered in response to the new start position, the experimenter waited until the unit firing adapted. The time variation between the two movements varied between 10 and $15 \mathrm{~s}$.

After the control session was imposed, the subject was informed of the nature of the movements to be imposed, cursive writing letters or numbers, and was told to focus on the movement trajectory described by the tip of the foot to recognize and name the writing movement. The same movements as those in the control session were then re-imposed. The subject was informed of the beginning and end of each movement with the words "go" and "stop." Each movement was imposed only once, and the subject was not informed as to whether he/she had succeeded in identifying the letter or number.

After these two experimental sessions, if the muscle spindle afferent activity persisted, some "control movements" were re-imposed once or several times to analyze the reproducibility of the unitary response. Twelve afferents were tested in this condition.

Sometimes, displacement of the electrode caused a recording loss before the whole set of movements was imposed in the recognition situation. In that case, the number of pairs of responses compared was less than six. The total number of pairs of responses compared was 135 for Ia afferents and 20 for group II afferents.

Data processing. The unitary afferent, EMG, and electrodermal activities, and the $x$ and $y$ displacements of the servocontrolled machine, were stored on a digital tape recorder (DTR 1802; Biologic, Claix, France). The data were processed off-line by means of Spike 2 software (Cambridge Electronic Design). Unitary action potentials were converted into TTL (transistor-transistor logic) pulses through a dual time/amplitude window discriminator (DDIS-1; BAK Electronics, Mount Airy, MD). The nerve spike events recorded were carefully inspected off-line on an expanded time scale.

A visual examination of the recordings showed some clear changes in the unit responses during recognition. To quantify these observations for the whole population of afferents, we first characterized the response of each afferent to each movement by determining the mean frequency and the variability of the instantaneous frequency (SD). This processing, however, did not yield different values even when the response showed a clear increase in the interspike interval variability (see Fig. 4). This finding is explained by the fact that large variations in the instantaneous discharge frequency are related to movement parameters. It was thus necessary to eliminate these variations to directly get to the small changes that seemed significant because they were related to the experimental conditions (i.e., instructions of not paying attention to or focusing on the movement).

For that reason, each unit response was filtered using a Hanning window (see Fig. $7 A, B$ ) created with the function in the signal processing toolbox from Matlab (7.0.1 under Windows OS; time constant, $300 \mathrm{~ms}$ ) i.e.,

$$
\omega(i)=\frac{1}{2}\left(1-\cos \left(2 \pi \frac{i}{n+1}\right)\right)
$$

where $i=1, \ldots n$.

Then, the variability of the instantaneous firing rate was characterized by an index, called "variability index" (VI), given by the following equation:

$$
V I=\sqrt{\frac{\sum_{i=1}^{n} R^{2}(i)}{n}},
$$

where $n$ is the total number of spikes in the Hanning window and $R$ is the residual vector (i.e., the difference between the original instantaneous firing rate values and the corresponding values produced by the Hanning filter).

\section{Results}

Muscle spindle afferent response during control session

Figure 2 shows, as an example, the activity of a muscle spindle primary ending from the EDL muscle recorded during a full control session in which six successive movements describing the shapes of letters were imposed to the foot. As can be seen, each letter gives rise to a typical and unique Ia discharge pattern, which has been described previously in detail (Roll et al., 2004). In brief, the Ia discharge is modulated in relation to the different parts of the movement, with each muscle stretching (plantar flexion), giving rise to an increased unit discharge and shortening, causing a silencing of the unit.

Most of the primary endings behaved in a similar way. The only minor difference observed for some Ia afferents was that 


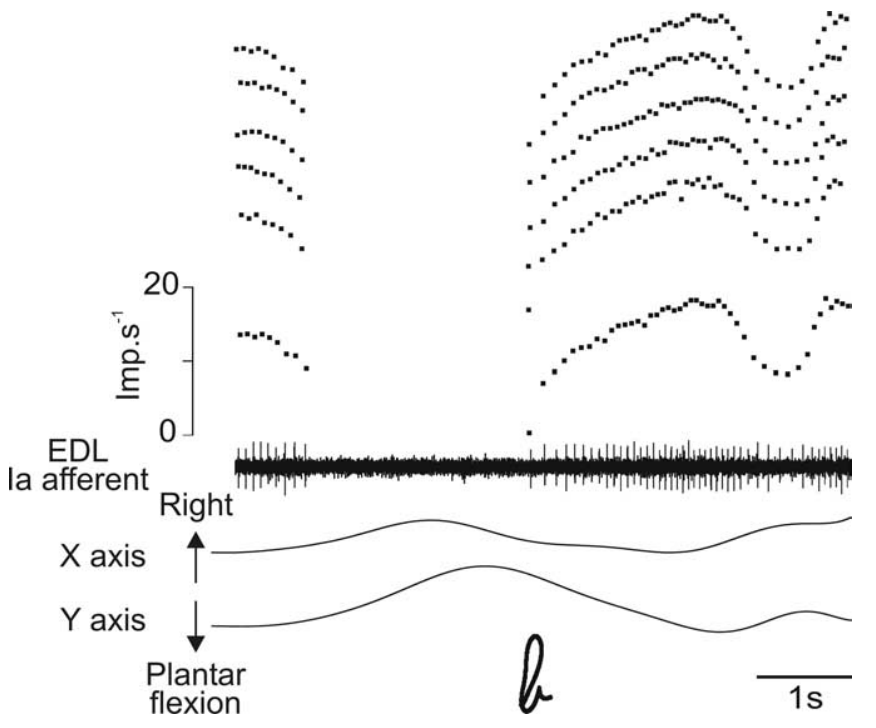

Figure 3. Reproducibility of the primary afferent responses to successive imposition of the same writing movement during control. Examples of responses of an la afferent belonging to the EDL muscle to six successive impositions of the movement forming the cursive letter "b." Represented from bottom to top are the $x$ and $y$ coordinates of the movement, the unitary activity with its corresponding instantaneous frequency curve, and the responses of the same afferent recorded during each of the five other successive movements illustrated by related instantaneous frequency curves. Imp, Impulse.

activity was more or less maintained during muscle shortening. Such a maintained and modulated activity was also observed with all of the tested secondary endings. Furthermore, the responses of all of the tested afferents originating from the same muscle were highly similar for each movement.

When the unitary recording was stable after both control and recognition sessions had been imposed, one or several control movements were re-imposed. In this case, the afferent responses were highly reproducible. This is illustrated by an example in Figure 3 that shows that the superimposed responses of an EDL Ia afferent to the letter "b," imposed six times, were fairly similar when the subject did not pay attention to the movements. The constancy of the response was also revealed by the VI, which ranged between 0.5 and 0.52 for the six movements, representing a maximum of $4 \%$ of variation of the lowest value. The reproducibility of the control responses was tested with 12 Ia afferents; the maximal VI variation was determined for each afferent and when averaged reached $8.5 \pm 6.5 \%$ (mean $\pm \mathrm{SD})$.

\section{Changes in muscle spindle afferent response when the subject} paid attention to the movement trajectory

Of the 26 primary endings tested, 15 (58\%) showed responses that differed for some movements when the subject was asked to focus on the movement trajectory described by the tip of the foot to recognize and name the writing movement. The changes were of two types. Figure 4 shows, as an example, the response of an Ia afferent belonging to the EDL muscle during the forming of the word "in." During the control session, the afferent showed increased activity during the plantar flexions and was silent during dorsal flexions. This pattern of activity was modified when the subject focused on the movement. The afferent became responsive throughout the entire trajectory of the movement, including the muscle shortening phases, which led to a reduction in the depth of modulation of discharge associated with movement. Moreover, the instantaneous frequency of discharge became much more irregular than the control. Such changes in the pat-
Table 1. Summary of the overall changes observed in the activity of 15 muscle spindle primary endings during recognition

\begin{tabular}{lllll}
\hline $\begin{array}{l}\text { Unit } \\
\text { number }\end{array}$ & $\begin{array}{l}\text { Triggered } \\
\text { spontaneous } \\
\text { activity }\end{array}$ & $\begin{array}{l}\text { Increased } \\
\text { interspike } \\
\text { interval variability }\end{array}$ & $\begin{array}{l}\text { Appearance } \\
\text { of doublets }\end{array}$ & $\begin{array}{l}\text { Decreased } \\
\text { dynamic } \\
\text { sensitivity }\end{array}$ \\
\hline 1 & $\mathrm{x}$ & $\mathrm{x}$ & $\mathrm{x}$ & 0 \\
2 & $\mathrm{x}$ & $\mathrm{x}$ & 0 & 0 \\
3 & 0 & 0 & 0 & $\mathrm{x}$ \\
4 & 0 & $\mathrm{x}$ & 0 & 0 \\
5 & 0 & $\mathrm{x}$ & $\mathrm{x}$ & 0 \\
6 & 0 & 0 & 0 & $\mathrm{x}$ \\
7 & 0 & 0 & 0 & $\mathrm{x}$ \\
8 & 0 & 0 & 0 & $\mathrm{x}$ \\
9 & 0 & 0 & 0 & $\mathrm{x}$ \\
10 & 0 & $\mathrm{x}$ & 0 & 0 \\
11 & 0 & 0 & 0 & $\mathrm{x}$ \\
12 & $\mathrm{x}$ & $\mathrm{x}$ & $\mathrm{x}$ & 0 \\
13 & $\mathrm{x}$ & $\mathrm{x}$ & $\mathrm{x}$ & 0 \\
14 & 0 & 0 & 0 & $\mathrm{x}$ \\
15 & 0 & 0 & 0 & $\mathrm{x}$ \\
\hline
\end{tabular}

$x$ and 0 stand for yes and no, respectively.
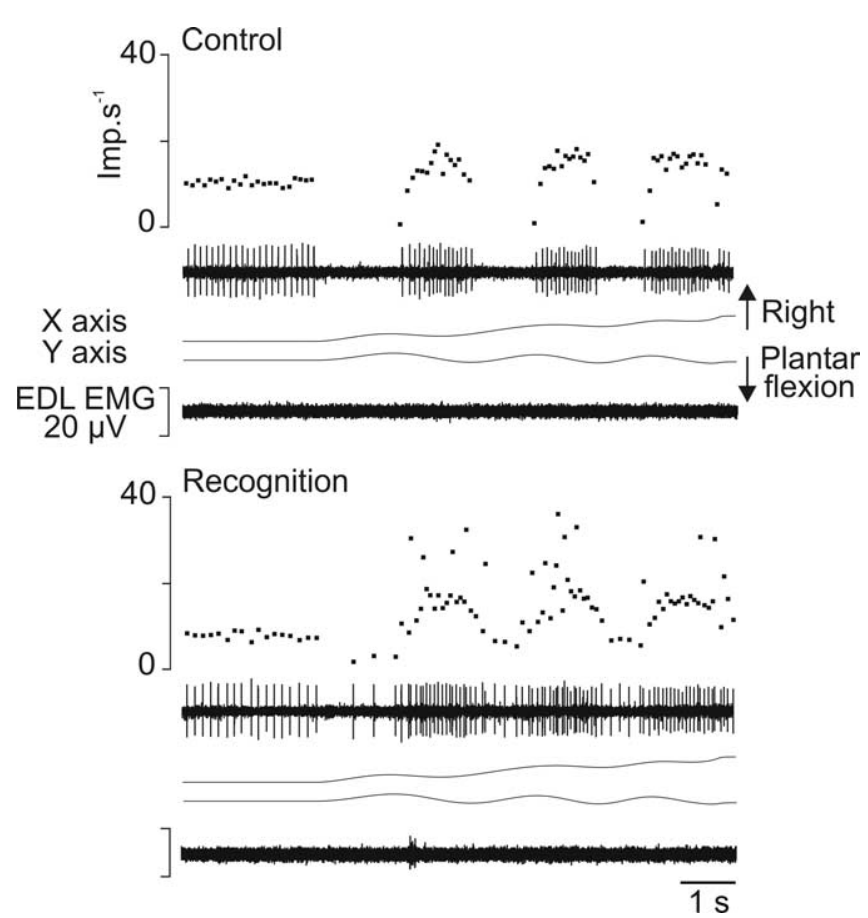

Figure 4. Example of the increased variability of the instantaneous frequency of la afferents during recognition compared with control. During control and recognition sessions (top and bottom part, respectively), the responses of an la afferent from the EDL muscle (unit 5) (Table 1) to imposed movement forming the word "in" are illustrated. Each part of the figure, from top to bottom, illustrates the instantaneous frequency curve and spike train, the imposed movement represented by its $x$ and $y$ coordinates, and EMG activity of the receptor-bearing muscle. Imp, Impulse.

tern of response were observed in seven Ia afferents (Table 1). The increased interspike interval variability was often associated with the appearance of doublets in the unit discharge (i.e., two spikes separated by an interval of $10-25 \mathrm{~ms}$ ). Finally, with four primary afferents, spontaneous activity sometimes appeared before the movement onset of recognition trials but not of control trials. The spontaneous Ia activity sometimes also became more irregular.

The second type of changes is illustrated in Figure 5, which shows the response of an Ia afferent belonging to the PL muscle 

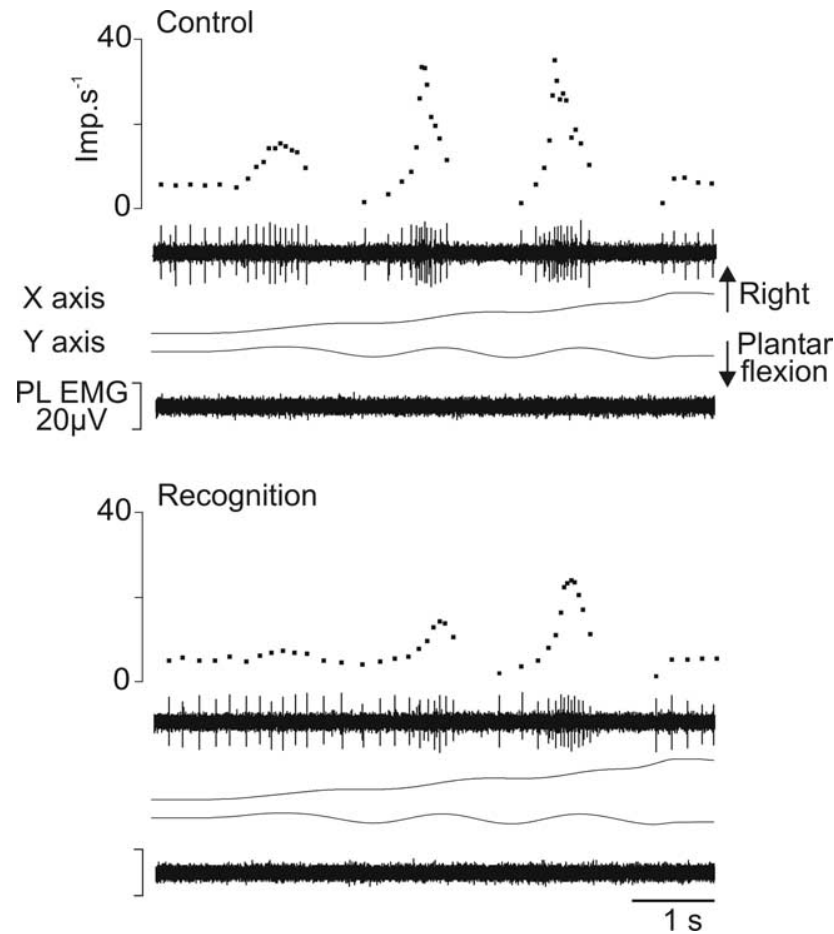

Figure 5. Example of the decreased dynamic sensitivity of muscle spindle primary endings during recognition compared with control. Same legend as in Figure 4, except that the la afferent (unit 3) (Table 1) rose from the PL muscle and that the trajectory of the imposed movements drew the letter " $m$. . Imp, Impulse.
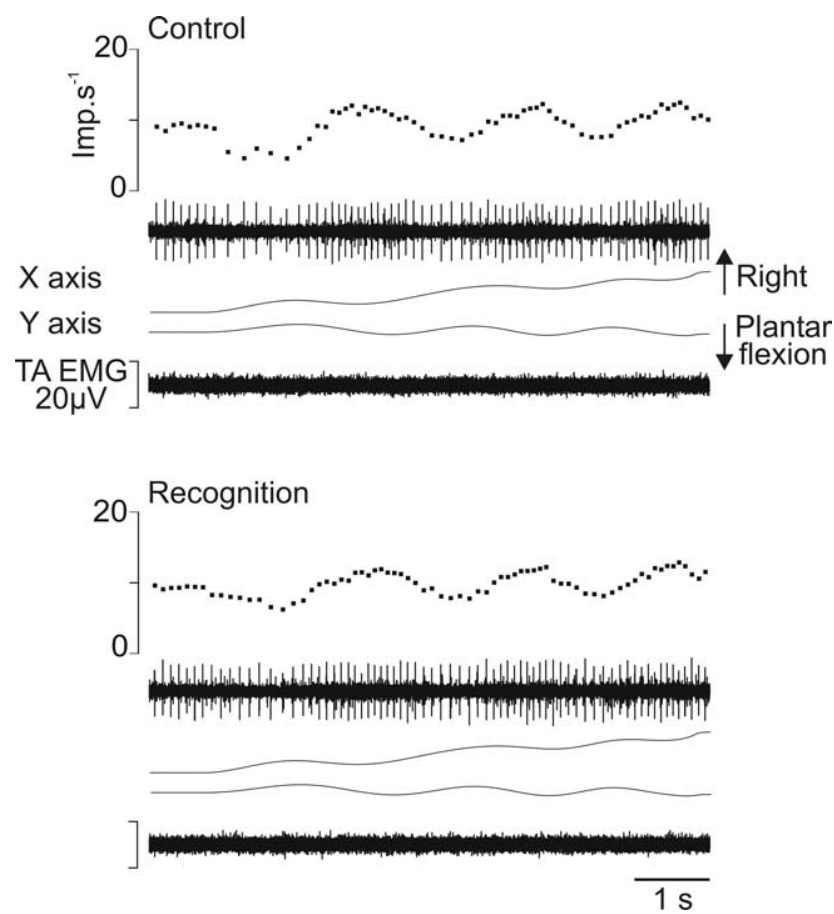

Figure 6. Example of slight changes in the response of group II afferents. Same legend as in Figure 4, except that the secondary afferent belonged to the TA muscle and the trajectory of the imposed movement drew the word "in." Imp, Impulse.

during the forming of the letter " $\mathrm{m}$." In the control session, the dorsal flexion movements were associated with bursts of activity corresponding to the three vertical elements of " $\mathrm{m}$," and plantar flexion movements were associated with silence. When the sub-
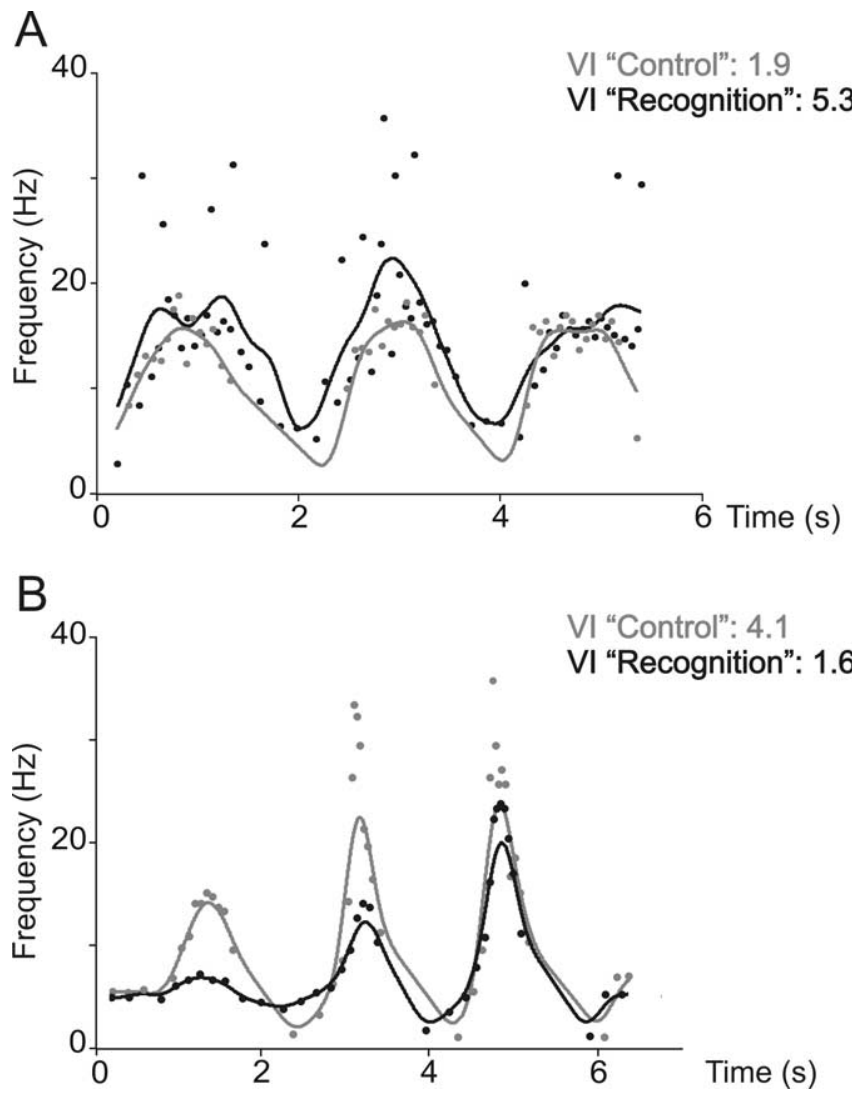

Figure 7. Characterization of the la afferent responses by the VI. $A$, The responses of the la afferent, previously shown on Figure 4, were filtered using a Hanning window (see Materials and Methods) during control (gray symbols and curve) and recognition (black symbols and curve). The VIs are given on the upper right part. Note that the VI increases during recognition. $\boldsymbol{B}$, Same as in $\boldsymbol{A}$, with the responses of la afferent previously shown in Figure 5 . Note that $\mathrm{VI}$ decreases during recognition.

ject focused on the movement to recognize it, the bursts of activity associated with muscle stretching at maximum velocities were considerably flattened for all three response components. Furthermore, the silent periods associated with muscle shortenings sometimes disappeared or decreased. Such a reduction in the depth of modulation of discharge associated with a decrease in the unit dynamic sensitivity was observed in eight Ia afferents. These afferents never showed changes in their spontaneous activity (Table 1).

The secondary afferents showed responses that were only slightly changed or unchanged compared with the control session. Figure 6 illustrates the response of one secondary ending arising from TA muscle during the forming of the word "in." All group II afferents behaved in a similar manner by regularly increasing and decreasing their activity during plantar flexion and dorsal flexion movements, respectively. This pattern of discharge might be slightly changed, as seen by the slight decrease in the depth of modulation of discharge during recognition in Figure 6.

The characterization of the responses of the whole population of afferents by the VI shows this index increased when the unit instantaneous discharge became more variable (Fig. 7A), and it decreased when the unit response exhibited a flattening of the bursts of discharge during recognition compared with control (Fig. 7B).

A response during recognition was considered as altered relative to control when the VI differed by $\geq 20 \%$, which represents three times the SD calculated in the control condition $(6.5 \%$, see 
paragraph above). Using this criterion, 22 Ia afferent responses presented an increased VI and 31 a decreased VI.

Given that the proprioceptive recognition task might be associated with changes in muscle spindle sensitivity, we wondered whether the success in recognition might be related to the occurrence of such changes. We therefore analyzed the number of correctly recognized movements among all of the possible ones (i.e., all movements excluding the trap "in," which could not be recognized because it was composed of two letters, whereas the subject was previously instructed that a single cursive letter would be imposed at a time). The percentage of correctly recognized movements amounted to $63 \%$ when changes in muscle spindle sensitivity were observed, but it was only $48 \%$ when muscle spindle sensitivity was unaltered.

\section{Discussion}

In a previous study, we showed that muscle spindle sensitivity to movement may be enhanced in completely relaxed subjects during the execution of a mental computation task (Ribot-Ciscar et al., 2000). That finding suggested that in humans, the CNS exerts a selective control of muscle spindle sensitivity via fusimotor neurons. Here, we tested whether the sensitivity of muscle spindle changes when the information from these receptors increases in importance, for example, in a task involving movement trajectory recognition in the absence of visual information, a situation that forces the subject to rely on proprioceptive cues only.

The results show that $58 \%$ of the tested muscle spindle primary endings presented a modified sensitivity to movement during recognition compared with the situation in which no attention was paid to the described trajectory. The response of the secondary afferents was only weakly changed or unchanged.

\section{Nature of the fusimotor effects}

The two types of effects on the sensitivity of muscle spindle primary endings may be related to two fusimotor effects. First, the decreased depth of modulation of discharge associated with increased activity during muscle shortenings and increased variability of the instantaneous frequency of discharge, which might also be concomitant with altered spontaneous activities, seem to be related to a static fusimotor drive (for review, see Hulliger, 1984; Prochazka, 1996). The effects observed, in particular the biasing effect, were neither as frequent nor as powerful as expected from animal data (Celichowski et al., 1994; Taylor et al., 1998; Durbaba et al., 2001). This difference may be explained by the natural condition of $\gamma$ driving in the present study compared with the electrical stimulation used in animal experiments. It has always been a common and intriguing observation that muscle spindle firing rates are much lower in humans than in animals (Prochazka and Hulliger, 1983), and the effect of the fusimotor system on muscle spindle sensitivity may also be weaker than in animals. This notion is supported, for example, by the fusimotorinduced increase in muscle afferent discharge during isometric contractions, which is 5-15 times lower in humans than in awake cats (Prochazka et al., 1977; Wilson et al., 1997).

Second, the decrease in the primary ending dynamic sensitivity may be interpreted as resulting from a decrease in dynamic fusimotor drive (for reviews, see Hulliger, 1984; Prochazka, 1996). This interpretation implies that a background of dynamic fusimotor activity was present during the control trials. Even if a $\gamma$-free condition was favored by the subjects' being relaxed during the control session, complete mental relaxation is not certain and some dynamic fusimotor activities may have been present.

That the few group II afferents tested showed only slight changes or no change in their responses to movements may appear surprising because the act of attention can trigger a static gamma drive, known to have a powerful effect on secondary endings in animals. However, first, the effects were not observed in all Ia tested afferents but in approximately half of the population; second, both altered/unaltered responses to consecutive movements were observed in the same experimental session. Thus, a fluctuation in the subject's attention level is probably a factor of importance in the present study, and we agree with Prochazka et al. (1992) suggesting that the fusimotor drive is largely dependent on the subject's internal attitude toward particular tasks and contexts. Consequently, that we only rarely observed small changes on group II afferents is possibly related to the small number of well identified secondary endings tested. Unfortunately, it is difficult to have more group II afferents; here, they represent $19 \%$ of the population of afferents recorded, which is similar to that in most microneurographic studies. Yet, it is also possible that we missed some of the effects. In the present study, the sensitivity of muscle spindle afferents was tested using imposed writing movements. These movements were chosen to involve the subject in a task that is more functionally relevant than the traditional ramp and hold movements. But, at the same time, we could not have access to the indexes classically used to characterize afferent sensitivity, such as dynamic index, static difference, and dynamic difference (Taylor et al., 1992). The VI we used appeared to be the most adequate for characterizing the changes in muscle afferent sensitivity. It is also possible, however, that this index was not as discriminating as the classical indexes to account for small changes in the responses. An example is seen in Figure 6 in which some decrease in the depth of modulation of discharge is observed in the response of the group II afferent during recognition, but this did not appear in the VI because it was 0.3 in both situations.

\section{The fusimotor-induced effects would facilitate proprioceptive coding of movements}

In a previous study, Vallbo and Al-Falahe (1990) reported that some Ia afferents exhibited slight increases in their responses when the subject's attention was directed to an imposed movement compared with when no attention was paid. These authors concluded that these changes did not present any functional relevance because the changes in muscle afferent activity were frequently associated with increased activity in the parent muscle. The presence of muscle activity in the passive condition was probably because of the instructions given, that is, to pay attention to the movement to actively reproduce it shortly afterward, as well as to the subjects' alternating passive and active movements.

In the present study, we showed that focusing attention on the movement may induce clear changes, in particular in Ia afferent discharge in completely relaxed subjects. We suggest that these fusimotor-induced effects facilitate proprioceptive coding when assisting in movement recognition. This is based on the higher chance of recognizing the movement when changes in muscle spindle sensitivity occurred, as found in the present study, but also on the following interpretations.

The observation of a depressed velocity component of the Ia response suggests that the coding of movement trajectory shape, which is the focused parameter of the task, is facilitated during recognition. Furthermore, the primary endings should become responsive throughout the whole trajectory of the movement during recognition, but only parts of the trajectory in control trials. This means that the preferred sensory sector of the afferent, 
previously defined as the ensemble of movement directions giving rise to an afferent response (Bergenheim et al., 2000), is enlarged. Consequently, the trajectory coding is probably facilitated and hence the recognition. Finally, the increased variability in the unit instantaneous frequency is also considered as optimizing proprioceptive information (Bergenheim et al., 1995; Tock et al., 2005). This increased variability is known to be the expression of a decorrelation, introduced by a working gamma system in parallel spindle afferents coming from the same muscle (Inbar et al., 1979). This decorrelation would be responsible for an enhanced quality of information transmitted by an ensemble of muscle afferents (Bergenheim et al., 1995; Tock et al., 2005). In that way, fusimotion is considered as a system using a stochastic resonance-type mechanism to increase muscle spindle sensitivity (Cordo et al., 1996; Fallon et al., 2004; for review, see Stein et al., 2005).

Interestingly, a uniform change in fusimotor activity (i.e., all spindles of a population are subjected to a similar $\gamma$ drive) was shown to decrease the ability of the ensemble to discriminate different muscle lengths in the cat (Pedersen et al., 1998). The interpretation of these results was that such a uniform fusimotor drive makes each muscle spindle response in an ensemble resemble the others. Consequently, the variability in the responses within a population is decreased, and this depresses the amount of information transmitted by the ensemble. The fact that focusing attention here induced changes in either static or dynamic fusimotor drive should be an additional means of increasing the variability in the Ia population response and thus the accuracy of muscle proprioceptive information.

In conclusion, involving a subject in a purely proprioceptive attention task may induce a fusimotor control of muscle spindle sensitivity independent of $\alpha$ activity. It is suggested that the consequence of this selective fusimotor drive might be to feed the brain movement trajectory information that is more accurate.

\section{References}

Albert F, Ribot-Ciscar E, Fiocchi M, Bergenheim M, Roll JP (2005) Proprioceptive feedback in humans expresses motor invariants during writing. Exp Brain Res 164:242-249.

Bergenheim M, Johansson H, Pedersen J (1995) The role of the gammasystem for improving information transmission in populations of Ia afferents. Neurosci Res 23:207-215.

Bergenheim M, Roll JP, Ribot-Ciscar E (1999) Microneurography in humans. In: Modern techniques in neuroscience research (Windhorst U, Johansson H, eds), pp 801-819. New York: Springer.

Bergenheim M, Ribot-Ciscar E, Roll JP (2000) Proprioceptive population coding of two-dimensional limb movements in humans: I. Muscle spindle feedback during spatially oriented movements. Exp Brain Res 134:301-310.

Celichowski J, Emonet-Denand F, Laporte Y, Petit J (1994) Distribution of static gamma axons in cat peroneus tertius spindles determined by exclusively physiological criteria. J Neurophysiol 71:722-732.

Cordo P, Inglis JT, Verschueren S, Collins JJ, Merfeld DM, Rosenblum S, Buckley S, Moss F (1996) Noise in human muscle spindles. Nature 383:769-770

Critchley HD (2002) Electrodermal responses: what happens in the brain. Neuroscientist 8:132-142.

Durbaba R, Taylor A, Ellaway PH, Rawlinson S (2001) Modulation of primary afferent discharge by dynamic and static gamma motor axons in cat muscle spindles in relation to the intrafusal fibre types activated. J Physiol (Lond) 532:563-574.

Edin BB, Vallbo AB (1990) Dynamic response of human muscle spindle afferents to stretch. J Neurophysiol 63:1297-1306.

Fallon JB, Carr RW, Morgan DL (2004) Stochastic resonance in muscle receptors. J Neurophysiol 91:2429-2436.

Gandevia SC, Burke D (1992) Does the nervous system depend on kines- thetic information to control natural limb movements? Behav Brain Sci 15:614-632.

Goodwin GM, McCloskey DI, Matthews PBC (1972) The contribution of muscle afferents to kinaesthesia shown by vibration induced illusions of movement and by the effects of paralysing joint afferents. Brain 95:705-748.

Hulliger M (1984) The mammalian muscle spindle and its central control. Rev Physiol Biochem Pharmacol 101:1-110.

Inbar G, Madrid J, Rudomin P (1979) The influence of the gamma system on cross-correlated activity of Ia muscle spindles and its relation to information transmission. Neurosci Lett 13:73-78.

Pedersen J, Ljubisavljevic M, Bergenheim M, Johansson H (1998) Alterations in information transmission in ensembles of primary muscle spindle afferents after muscle fatigue in heteronymous muscle. Neuroscience 84:953-959.

Prochazka A (1996) Proprioceptive feedback and movement regulation. In: Handbook of physiology, Sec 12. Exercise: regulation and integration of multiple systems (Rowell L, Sheperd JT, eds), pp 89-127. New York: American Physiological Society.

Prochazka A, Hulliger M (1983) Muscle afferent function and its significance for motor control mechanisms during voluntary movements in cat, monkey, and man. In: Motor control mechanisms in health and disease (Desmedt JE, ed), pp 93-132. New York: Raven.

Prochazka A, Westerman RA, Ziccone SP (1977) Ia afferent activity during a variety of voluntary movements in the cat. J Physiol (Lond) 268:423-448.

Prochazka A, Hulliger M, Zangger P, Appenteng K (1985) 'Fusimotor set': new evidence for alpha-independent control of gamma-motoneurones during movement in the awake cat. Brain Res 339:136-140.

Prochazka A, Gorassini MT, Taylor J (1992) Adaptative control of proprioception. In: Muscle afferents and spinal control of movement (Jami L, Pierrot-Deseilligny E, Zytnicki D, eds), pp 129-136. Oxford: Pergamon.

Ribot E, Roll JP, Vedel JP (1986) Efferent discharges recorded from single skeletomotor and fusimotor fibres in man. J Physiol (Lond) 375:251-268.

Ribot-Ciscar E, Rossi-Durand C, Roll JP (2000) Increased muscle spindle sensitivity to movement during reinforcement manoeuvres in relaxed human subjects. J Physiol (Lond) 523:271-282.

Roll JP, Vedel JP (1982) Kinaesthetic role of muscle afferents in man, studied by tendon vibration and microneurography. Exp Brain Res 47:177-190.

Roll JP, Vedel JP, Ribot E (1989) Alteration of proprioceptive messages induced by tendon vibration in man: a microneurographic study. Exp Brain Res 76:213-222.

Roll JP, Albert F, Ribot-Ciscar E, Bergenheim M (2004) "Proprioceptive signature" of cursive writing in humans: a multi-population coding. Exp Brain Res 157:359-368.

Stein RB, Gossen ER, Jones KE (2005) Neuronal variability: noise or part of the signal? Nature Rev Neurosci 6:389-397.

Taylor A, Durbaba R, Rodgers JF (1992) The classification of afferents from muscle spindles of the jaw-closing muscles of the cat. J Physiol (Lond) 456:609-628.

Taylor A, Ellaway PH, Durbaba R (1998) Physiological signs of the activation of bag2 and chain intrafusal muscle fibers of gastrocnemius muscle spindles in the cat. J Neurophysiol 80:130-142.

Taylor A, Ellaway PH, Durbaba R, Rawlinson S (2000) Distinctive patterns of static and dynamic gamma motor activity during locomotion in the decerebrate cat. J Physiol (Lond) 529:825-836.

Tock Y, Inbar GF, Steinberg Y, Ljubisavljevic M, Thunberg J, Windhorst U, Johansson H (2005) Estimation of muscle spindle information rate by pattern matching and the effect of gamma system activity on parallel spindles. Biol Cybern 92:316-332.

Vallbo AB, Al-Falahe NA (1990) Human muscle spindle response in a motor learning task. J Physiol (Lond) 421:553-568.

Vallbo AB, Hagbarth KE (1968) Activity from skin mechanoreceptors recorded percutaneously in awake human subjects. Exp Neurol 21:270-289.

Vallbo AB, Hagbarth KE, Torebjork HE, Wallin BG (1979) Somatosensory, proprioceptive, and sympathetic activity in human peripheral nerves. Physiol Rev 59:919-957.

Wilson LR, Gandevia SC, Burke D (1997) Discharge of human muscle spindle afferents innervating ankle dorsiflexors during target isometric contractions. J Physiol (Lond) 504:221-232. 